\title{
Impact of the PROFHER trial findings on surgeons' clinical practice
}

\author{
AN ONLINE QUESTIONNAIRE SURVEY
}

\section{Jefferson, S. Brealey, H. Handoll, A. Keding, L. Kottam, I. Sbizzera, A. Rangan}

York Trials Unit, Department of Health Sciences, University of York, Heslington, York, United Kingdom

L. Jefferson, BSc, MSc, PhD, Research Fellow, Department of Health Sciences, University of York, Heslington, York YO10 5DD, UK. - S. Brealey, BSc, PhD, Research Fellow, York Trials Unit, University of York,

A. Keding, BSc, MSc, Lecturer in Statistics, York Trials Unit, University of York,

I. Sbizzera, BSc, MSc, Trainee Statistician, York Trials Unit, University of York, Lower Ground Floor, ARRC Building, Heslington, York YO10 5DD, UK.

H. Handoll, DPhil, Reader in Orthopaedics, School of Health and Social Care, Teesside University, Middlesbrough, Tees Valley TS1 3BA, UK.

L. Kottam, BSc, MSc, MSc, PhD Career Scientist, Orthopaedics,

Department of Trauma and

Orthopaedics, South Tees

Hospitals NHS Trust, James Cook University Hospital, Marton Road, Middlesborough, Tees Valley TS4 3BW, UK.

A. Rangan, ChM, FRCS(Tr\&Orth), Professor of Orthopaedic Surgery, York Trials Unit, University of York, Lower Ground Floor, ARRC Building, Heslington, York YO10 5DD, UK and School of Health and Social Care,

Middlesbrough, Tees Valley TS1 3BA UK and South Tees Hospitals NHS Trust, Marton Road, Middlesborough TS4 3BW, UK.

Correspondence should be sent to S. Brealey; email: stephen.brealey@ york.ac.uk

doi: $10.1302 / 2046-3758.610$ BJR-2017-0170

Bone loint Res 2017;6:590-599. Received: 20 June 2017;

Accepted: 25 August 2017

\section{Objectives}

To explore whether orthopaedic surgeons have adopted the Proximal Fracture of the Humerus: Evaluation by Randomisation (PROFHER) trial results routinely into clinical practice.

\section{Methods}

A questionnaire was piloted with six orthopaedic surgeons using a 'think aloud' process. The final questionnaire contained 29 items and was distributed online to surgeon members of the British Orthopaedic Association and British Elbow and Shoulder Society. Descriptive statistics summarised the sample characteristics and fracture treatment of respondents overall, and grouped them by whether they changed practice based on PROFHER trial findings. Freetext responses were analysed qualitatively for emerging themes using Framework Analysis principles.

\section{Results}

There were complete responses from 265 orthopaedic and trauma surgeons who treat patients with proximal humeral fractures. Around half (137) had changed practice to various extents because of PROFHER, by operating on fewer PROFHER-eligible fractures. A third (43) of the 128 respondents who had not changed practice were already managing patients non-operatively. Those who changed practice were more likely to be younger, work in a trauma unit rather than a major trauma centre, be specialist shoulder surgeons and treat fewer PROFHER-eligible fractures surgically. This group gave higher scores when assessing validity and applicability of PROFHER. In contrast, a quarter of the non-changers were critical, sometimes emphatically, of PROFHER. The strongest theme that emerged overall was the endorsement of evidence-based practice.

\section{Conclusion}

PROFHER has had an impact on surgeons' clinical practice, both through changing it, and through underpinning existing non-operative practice. Although some respondents expressed reservations about the trial, evidence from such trials was found to be the most important influence on surgeons' decisions to change practice.

Cite this article: Bone Joint Res 2017;6:590-599.

Keywords: Proximal humeral fractures, Surgery, Non-operative care, Survey, Surgeons

\section{Article focus}

- Large, publicly-funded, pragmatic multicentre trials such as the Proximal Fracture of the Humerus: Evaluation by Randomisation (PROFHER) trial involve very substantial investment. It is important to determine the impact of such endeavours.

- This article reports the findings of an online survey completed by orthopaedic and trauma surgeons who treat patients with proximal humeral fractures in the United Kingdom.

- The survey explored whether these surgeons had adopted the PROFHER trial results routinely into practice.

\section{Key messages}

- The survey showed that around half of the 265 respondents had changed practice because of PROFHER, with a third of those who had not changed 
already managing patients eligible for PROFHER non-operatively.

- Qualitative analysis provided a more complex picture of the impact from PROFHER, such as in the use of evidence to underpin non-operative decisions.

- The strongest overall message was the endorsement of evidence-based practice.

\section{Strengths and limitations}

- The survey obtained often detailed responses from a substantial number of orthopaedic surgeons who currently treat patients with fractures of the proximal humerus in the United Kingdom.

- The actual response rate could not be calculated. Nonetheless, some evidence was available that provided reassurance in terms of adequate participation and low response bias.

- The linked qualitative analysis informed and greatly enriched the interpretation of the quantitative data.

\section{Introduction}

The Proximal Fracture of the Humerus: Evaluation by Randomisation (PROFHER) trial was a publicly funded, pragmatic, multicentre randomised controlled trial (RCT) that compared surgical treatment with non-surgical treatment for the majority of adults with displaced proximal humeral fractures involving the surgical neck. ${ }^{1}$ The trial inclusion criteria are shown in Figure 1. Between September 2008 and April 2011, 250 patients were recruited from 32 NHS hospitals in the United Kingdom. The main follow-up report, which was published in March 2015, showed no benefit of surgery over a twoyear follow-up, when compared with non-surgical treatment for the study-defined fracture population. ${ }^{1}$ The study also showed significantly greater costs associated with surgical management. ${ }^{2}$ These findings have been incorporated into a guideline published in 2016 by the National Institute for Health and Care Excellence (NICE), which recommends that for adults "with displaced low energy proximal humerus fractures", "non-surgical management for definitive treatment of uncomplicated injuries" should be offered. ${ }^{3}$

Reflecting an ageing population, there is an increasing incidence of proximal humeral fractures, ${ }^{4}$ which already account for $5 \%$ to $6 \%$ of all adult fractures. ${ }^{5}$ The increasing but non-evidenced trend for surgical treatment, ${ }^{6}$ with an associated increase in direct treatment costs, ${ }^{7}$ and, crucially, an acknowledgement of collective equipoise by a substantial number of orthopaedic surgeons involved in treating these injuries, provided a suitable context for conducting PROFHER. Overall, PROFHER involved a substantial investment in terms of funding through the National Institute of Health Research (NIHR) and through contributions from hundreds of people, including surgeons and other clinicians at the participating sites. Given this substantial investment in research, the incorporation of research findings into NICE guidance and the continuing burden of these injuries on individuals and society, it is pertinent to explore whether, and in what way, the results of the study have affected surgeon decision-making.

Thus, we undertook a survey of orthopaedic surgeons with the primary objective of exploring whether there was awareness of the PROFHER trial results and whether these findings have been adopted routinely into clinical practice. This included identifying surgeons' treatment of these patients and the potential reasons for these treatment choices. In addition, we collected surgeons' views about other factors, including the reconfiguration of trauma services in the United Kingdom beginning in 2012 that may have influenced surgical practice more generally.

\section{Materials and Methods}

Participants. In September 2016, surgeons were invited to participate in an online questionnaire via membership emails through the British Orthopaedic Association (BOA) and British Elbow and Shoulder Society (BESS). The email asked members to complete the questionnaire once, and only if they "currently treat patients for a proximal humerus fracture". A reminder email was sent to potential participants after two weeks. In order to improve response rates, participants were offered the opportunity to enter into a prize draw to win a $£ 100$ gift voucher upon questionnaire completion. A winner was chosen at random two weeks following closure of the online questionnaire.

Ethical approval was gained from the University of York Department of Health Sciences Research Governance Committee and permissions from BOA and BESS research committees before disseminating the survey. Surgeons were not obliged to take part in this study and were given the opportunity to withdraw at any time during completion of the online questionnaire.

Questionnaire. Questionnaire items were generated through discussion between authors, which included expert researchers and a consultant orthopaedic surgeon who worked together on the PROFHER trial. The questionnaire draft was then piloted with a sample of six orthopaedic surgeons (members of BESS) in order to test the feasibility, ease of completion and face validity of questionnaire items prior to wider dissemination. A 'think aloud' process was undertaken, ${ }^{8}$ whereby each surgeon was asked to complete the questionnaire and talk through their responses with a researcher (LK) as they completed each item. By surgeons voicing their thought processes and meanings attributed to each question, the validity of the questionnaire items was tested to see how well these addressed the study objectives. This stage of the questionnaire testing also helped to identify any important omissions from the questionnaire items, and to record the length of time taken to complete the questionnaire. 
Inclusion criteria

Adults (aged 16 or above) presenting within three weeks of their injury with a radiologically confirmed displaced fracture of the humerus involving the surgical neck. This should include all two part surgical neck fractures; three part (including surgical neck) and four part fractures of proximal humerus (Neer Classification).

It may also include displaced surgical neck fractures that do not meet the exact displacement criteria of the Neer Classification ( $1 \mathrm{~cm}$ and/or $45^{\circ}$ angulation of displaced parts) where this reflects an individual surgeon's uncertainty (e.g. whether or not the surgical neck fracture should be treated surgically).

Exclusion criteria

Associated dislocation of the injured joint of the shoulder

Open fracture

Mentally incompetent patient: unable to understand trial procedure or instructions for rehabilitation; significant mental impairment that would preclude compliance with rehabilitation and treatment advice

Comorbidities precluding surgery/anaesthesia

A clear indication for surgery such as severe soft-tissue compromise requiring surgery/emergency treatment (nerve injury/dysfunction)

Multiple injuries: same limb fractures; other upper limb fractures

Pathological fractures (other than osteoporotic) and terminal illness

Participant not resident in catchment area of trauma centre

Proximal Fracture of the Humerus: Evaluation by Randomisation trial inclusion and exclusion criteria.

The final questionnaire contained 29 items (summarised in Fig. 2) and was converted into electronic format using Qualtrics software (Provo, Utah). The survey, which took approximately ten minutes to complete, consisted of four sections. Sections 1 and 2 identified respondent demographics, experience levels and level of engagement in research generally. Section 3 explored in turn: respondents' current surgical practice in relation to all acute proximal humeral fractures, respondents' current treatment of patients with acute "low energy uncomplicated displaced fractures of the proximal humerus involving the surgical neck" and how much influence the PROFHER findings had on their practice. A final and optional section explored respondents' general thoughts on what may influence changes to surgical practice. In order to improve usability and reduce the time taken to complete the questionnaire, skip logic was used throughout, so that certain items only appeared based on participants' previous item responses. A progress bar, previous page facility, and 'save and resume later' function were also included to aid usability.

Statistical analysis. Descriptive statistics were used to summarise the characteristics of the sample (Sections 1 and 2), in total and grouped by whether participants did or did not change practice based on the PROFHER findings. Quantitative responses regarding current and changed treatment practices (Section 3) were summarised descriptively in total and grouped by selected sample characteristics (including surgical and research experience).
Qualitative analysis. Free-text responses were analysed qualitatively using principles of Framework Analysis, ${ }^{9}$ whereby key concepts and recurrent themes were generated through an iterative process of familiarisation with the data, coding, and recoding until a thematic framework emerged. Three researchers (LJ, HH and SB) with expertise in different areas (qualitative research, orthopaedic research and surgical trial design) coded the data with regular discussion. Due to the large amount of qualitative data yielded, numerical counting of themes occurring in participants' responses helped to explore trends.

\section{Results}

In total, 317 surgeons accessed the online questionnaire, which was sent out indiscriminately to an estimated 2600 surgeon members, some overlapping, of BOA and BESS. It is not possible to calculate a response rate, as respondents were asked to complete the questionnaire only if they were a practising surgeon who was currently treating patients with proximal humerus fractures. Of 317 respondents, 285 (89.9\%) completed Section 1 and at least part of Section 2 and Section 3. The remaining 32 (10.1\%) survey entries were excluded, as none of the questions had been completed $(n=29)$ or only those in Section 1 (basic demographics, $n=3$ ).

The study's prime question of interest ("Have you changed your practice within the last couple of years in terms of surgical treatment for these fractures?") was answered (Yes/No) in 265 questionnaires (93.0\% of 285); these were therefore included in the main analysis. The 
Section 1: About you

- Age

- Gender

- Where are you based?

- Where do you work?

- Which of the following best describes your role?

- What clinical post do you currently hold?

- How many years experience do you have of treating patients with fractures of the proximal humerus?

\section{Section 2: Engagement in clinical trials research}

- Have you been a grant holder, Principal Investigator or held any other substantive role (e.g. Trial Steering Group member) for a NIHR randomised controlled surgical trial?

- Did you participate in the PROFHER trial?

- If yes, were you a Principal Investigator for a PROFHER site?

- Before reading the research summary above, were you aware of the PROFHER findings?

- If yes, where did you hear about the results? (please tick all that apply)

- Also, if yes, on the following scale of 0 (not at all valid) to 10 (extremely valid), please indicate how valid you found the results of the PROFHER Trial?

- Also, if yes, on the following scale of 0 (not at all applicable) to 10 (extremely applicable), please indicate how much you consider the results apply to your clinical practice for the type of fractures included in PROFHER?

Section 3: Treatment of acute displaced proximal humeral fractures

- Please estimate as accurately as possible, how many proximal humeral fractures (all types) you operated on during the last financial year (between April 2015 and March 2016)?

- Please indicate the surgical methods you used (please tick all that apply)

- Please estimate as accurately as possible, how many of the fractures you operated on were acute 'uncomplicated' displaced fractures of the proximal humerus involving the surgical neck (i.e. eligible for the PROFHER trial)?

- Have you changed your practice within the last couple of years in terms of surgical treatment for patients with fractures eligible for the PROFHER trial?

- If No, please briefly describe why your practice has remained the same

- If Yes, please describe briefly how your practice has changed

- Also if Yes, was this because you became aware of the PROFHER findings?

- If Yes, please describe how PROFHER findings prompted this change?

- If no or partly, please describe why PROFHER findings did not change your practice?

- To what extent do the cost-effectiveness findings of PROFHER have an impact on your decision to operate?

- Have there been any other external changes, such as the changes to the delivery of trauma services, that have impacted on your current practice for these fractures?

(Optional) Section 4: General thoughts on changing surgical practice

- In your opinion, what factors are likely to influence your decision to change surgical practice?

- In your opinion, is practicing Evidenced Based Medicine becoming the norm in orthopaedic surgery?

- What could your specialist society (e.g. BESS, the British Orthopaedic Association or Royal College of Surgeons) do to guide any change in your practice?

- If you have any further comments you would like to make then please provide them here

Fig. 2

Questionnaire items for this study (NIHR, National Institute for Health Research; PROFHER, Proximal Fracture of the Humerus: Evaluation by Randomisation; BESS, British Elbow and Shoulder Society).

20 participants who did not answer this question tended to be younger, less likely to be specialised in trauma or shoulder surgery, and have fewer years of experience.

Participant characteristics. Table I shows that most participants were aged between 36 and 55 years old
(70.2\%), were male (94.0\%), worked in England (83.6\%) and worked in a trauma unit (65.0\%). Just over half were specialised shoulder surgeons (54.3\%), mainly of consultant grade (82.9\%) and with over ten years of experience in treating proximal humeral fractures (59.6\%). 
Table I. Characteristics of participants who did/did not change practice because of the Proximal Fracture of the Humerus: Evaluation by Randomisation trial. Missing responses are excluded from all percentage calculations

\begin{tabular}{|c|c|c|c|}
\hline Characteristic & $\begin{array}{l}\text { Changed practice } \\
(n=137)\end{array}$ & $\begin{array}{l}\text { Did not change } \\
\text { practice }(n=128)\end{array}$ & Total $(n=265)$ \\
\hline \multicolumn{4}{|l|}{ Age (yrs), n (\%) } \\
\hline$\leqslant 35$ & $17(12.4)$ & $6(4.7)$ & $23(8.7)$ \\
\hline 36 to 45 & $52(38.0)$ & $46(35.9)$ & $98(37.0)$ \\
\hline 46 to 55 & $44(32.1)$ & $44(34.4)$ & $88(33.2)$ \\
\hline 56 to 65 & $23(16.8)$ & $31(24.2)$ & $54(20.4)$ \\
\hline$\geqslant 66$ & $1(0.7)$ & $1(0.8)$ & $2(0.8)$ \\
\hline Missing & 0 & 0 & 0 \\
\hline \multicolumn{4}{|l|}{ Gender, n (\%) } \\
\hline Male & $126(92.0)$ & $123(96.1)$ & $249(94.0)$ \\
\hline Female & $11(8.0)$ & $5(3.9)$ & $16(6.0)$ \\
\hline Missing & 0 & 0 & 0 \\
\hline \multicolumn{4}{|l|}{ Country of work, n (\%) } \\
\hline England & $112(81.8)$ & $107(85.6)$ & $219(83.6)$ \\
\hline Scotland & $9(6.6)$ & $7(5.6)$ & $16(6.1)$ \\
\hline Wales & $10(7.3)$ & $4(3.2)$ & $14(5.3)$ \\
\hline Northern Ireland & $4(2.9)$ & $6(4.8)$ & $10(3.8)$ \\
\hline Other $^{*}$ & $2(1.5)$ & $1(0.8)$ & $3(1.1)$ \\
\hline Missing & 0 & 3 & 3 \\
\hline \multicolumn{4}{|l|}{ Place of work, n (\%) } \\
\hline Major Trauma Centre (England) or equivalent tertiary hospital & $28(20.4)$ & $37(29.4)$ & $65(24.7)$ \\
\hline Trauma Unit (England) or equivalent secondary care hospital & $96(70.1)$ & $75(59.5)$ & $171(65.0)$ \\
\hline District General Hospital & $8(5.8)$ & $9(7.1)$ & $17(6.5)$ \\
\hline Other $^{\dagger}$ & $5(3.6)$ & $5(4.0)$ & $10(3.8)$ \\
\hline Missing & 0 & 2 & 2 \\
\hline \multicolumn{4}{|l|}{ Role, n (\%) } \\
\hline General orthopaedic surgeon & $48(35.0)$ & $46(35.9)$ & $94(35.5)$ \\
\hline Trauma surgeon & $10(7.3)$ & $17(13.3)$ & $27(10.2)$ \\
\hline Shoulder surgeon & $79(57.7)$ & $65(50.8)$ & $144(54.3)$ \\
\hline Missing & 0 & 0 & 0 \\
\hline \multicolumn{4}{|l|}{ Clinical post held, n (\%) } \\
\hline Consultant & $111(81.6)$ & $107(84.3)$ & $218(82.9)$ \\
\hline Specialty Trainee, ST7 or ST8 & $10(7.4)$ & $8(6.3)$ & $18(6.8)$ \\
\hline Specialty Trainee, ST1 to ST6 & $7(5.1)$ & $4(3.1)$ & $11(4.2)$ \\
\hline Staff and Associate specialists & $4(2.9)$ & $6(4.7)$ & $10(3.8)$ \\
\hline Fellows & $4(2.9)$ & $2(1.6)$ & $6(2.3)$ \\
\hline Missing & 1 & 1 & 2 \\
\hline \multicolumn{4}{|c|}{ Years of experience in treating fractures of the proximal humerus, $\mathbf{n}(\%)$} \\
\hline 0 to 5 & 15 (10.9) & $10(7.8)$ & $25(9.4)$ \\
\hline 6 to 10 & $49(35.8)$ & $33(25.8)$ & $82(30.9)$ \\
\hline 11 to 15 & $26(19.0)$ & $31(24.2)$ & $57(21.5)$ \\
\hline 16 to 20 & 19 (13.9) & $22(17.2)$ & $41(15.5)$ \\
\hline$\geqslant 21$ & $28(20.4)$ & $32(25.0)$ & $60(22.6)$ \\
\hline Missing & 0 & 0 & 0 \\
\hline
\end{tabular}

*the three respondents who chose 'Other' for this question worked in France, Singapore and the United States, respectively

¡‘Other' here referred, for example, to private centres and university hospitals

Survey results. Of the 265 participants included in the analysis, $137(51.7 \%)$ had changed their practice in terms of surgical treatment of acute displaced proximal humeral fractures entirely (90 responses) or partly (47 responses) because of the PROFHER findings. In comparison, 128 participants (48.3\%) did not change practice because of PROFHER, although three had changed practice for other reasons. However, of these 128 respondents, free-text responses revealed that 43 surgeons were already managing these types of patients nonoperatively; for example: "I already practised along the lines of the PROFHER findings".

Comparison of participants who did or did not change practice because of PROFHER. Surgeons who changed practice tended to be slightly younger, working in a trauma unit rather than a major trauma centre, be a shoulder surgeon rather than a trauma surgeon and have fewer years of experience in treating these fractures (Table I).

Table II summarises the clinicians' prior involvement in research, direct involvement with the PROFHER trial and perceived validity and applicability of the PROFHER findings. Surgeons who changed their practice were more likely to have had a substantive role, mainly as Principal Investigator (PI), in an NIHR randomised surgical trial in the past (19.7\% versus $8.7 \%)$. Overall, 39 participants (14.7\%) had participated in the PROFHER trial, nine as Pls. Participation did not appear to be related to subsequent change in practice.

The majority of participants $(245 ; 92.5 \%)$ were aware of the PROFHER findings before reading the research 
Table II. Research engagement of participants who did/did not change practice because of the Proximal Fracture of the Humerus: Evaluation by Randomisation (PROFHER). Missing responses are excluded from all percentage calculations

\begin{tabular}{|c|c|c|c|}
\hline & $\begin{array}{l}\text { Changed practice } \\
(n=137)\end{array}$ & $\begin{array}{l}\text { Did not change practice } \\
(n=128)\end{array}$ & Total $(n=265)$ \\
\hline \multicolumn{4}{|c|}{ Substantive role in an NIHR randomised controlled surgical trial, n (\%) } \\
\hline Yes & $27(19.7)$ & $11(8.7)$ & $38(14.3)$ \\
\hline No & $110(80.3)$ & $116(91.3)$ & $226(85.6)$ \\
\hline Missing & 0 & 1 & 1 \\
\hline \multicolumn{4}{|l|}{ Role held, $n(\%)^{* \dagger}$} \\
\hline Principal Investigator (PI) & $21(91.3)$ & $8(88.9)$ & $29(90.6)$ \\
\hline Chief Investigator & $1(4.3)$ & $0(0.0)$ & $1(3.1)$ \\
\hline Co-applicant & $2(8.7)$ & $0(0.0)$ & $2(6.3)$ \\
\hline Member of oversight committee & $3(13.0)$ & $1(11.1)$ & $4(12.5)$ \\
\hline Missing & 4 & 2 & 6 \\
\hline \multicolumn{4}{|l|}{ Participation in the PROFHER trial, n (\%) } \\
\hline Yes & $22(16.1)$ & $17(13.3)$ & $39(14.7)$ \\
\hline No & 115 (83.9) & $111(86.7)$ & $226(85.3)$ \\
\hline Missing & 0 & 0 & 0 \\
\hline \multicolumn{4}{|l|}{ PI for a PROFHER site, $\mathrm{n}(\%)^{\ddagger}$} \\
\hline Yes & $5(22.7)$ & $4(23.5)$ & $9(23.1)$ \\
\hline No & $17(77.3)$ & $13(76.5)$ & $30(76.9)$ \\
\hline Missing & 0 & 0 & 0 \\
\hline \multicolumn{4}{|l|}{ Awareness of the PROFHER findings, $\mathrm{n}(\%)$} \\
\hline Yes & $134(97.8)$ & $111(86.7)$ & $245(92.5)$ \\
\hline No & $3(2.2)$ & $17(13.3)$ & $20(7.5)$ \\
\hline Missing & 0 & 0 & 0 \\
\hline \multicolumn{4}{|l|}{ Where heard about PROFHER, $\mathrm{n}(\%)^{*} \S$} \\
\hline Attended a meeting where the results of PROFHER were presented & 105 (78.4) & $70(63.1)$ & $175(71.4)$ \\
\hline Read one or more of the research publications & $94(70.1)$ & $77(69.4)$ & $171(69.8)$ \\
\hline Through working at a participating site & $19(14.2)$ & $15(13.5)$ & $34(13.9)$ \\
\hline Local contacts & $38(28.4)$ & $26(23.4)$ & $64(26.1)$ \\
\hline NICE guidelines & $19(14.2)$ & $13(11.7)$ & $32(13.1)$ \\
\hline Missing & 0 & 0 & 0 \\
\hline \multicolumn{4}{|l|}{ Validity of PROFHER findings (0 to 10)* } \\
\hline Mean (SD) & $7.4(1.7)$ & $5.3(2.4)$ & $6.4(2.3)$ \\
\hline Median (range) & $7(0$ to 10$)$ & $6(0$ to 10$)$ & $7(0$ to 10$)$ \\
\hline Missing & 0 & 0 & 0 \\
\hline \multicolumn{4}{|l|}{ Applicability of PROFHER findings to own practice $(0 \text { to } 10)^{*}$} \\
\hline Mean (SD) & $7.6(1.7)$ & $5.6(2.6)$ & $6.7(2.4)$ \\
\hline Median (range) & $8(1$ to 10$)$ & $6(0$ to 10$)$ & $7(0$ to 10$)$ \\
\hline Missing & 0 & 0 & 0 \\
\hline
\end{tabular}

*more than one response was possible for this question

this total is based on the 38 participants who had a substantive role in a NIHR randomised controlled surgical trial

\#this total is based on the 39 participants who had participated in the PROFHER trial

sthis total is based on the 245 participants who were aware of the PROFHER findings; 111 changed their practice because of PROFHER; 112 did not change

their practice

NIHR, National Institute for Health Research; NICE, National Institute for Health and Care Excellence

summary reported in the first page of the survey. Awareness was less in the group who did not change practice $(86.7 \%)$. Curiously, three participants who reported to have changed practice because of PROFHER reported not to have been aware of it. Most participants heard about the trial results in meetings where these were presented $(71.1 \%)$ and/or read them in one or more research publication (69.8\%). Participants who changed their practice were more likely to have attended a meeting (78.4\% versus $62.5 \%)$.

Participants who changed their surgical practice gave higher ratings (out of 10) to the validity of the PROFHER results (mean 7.4 versus 5.3 ) and the applicability of results to their own practice (mean 7.6 versus 5.6) than participants who did not change practice. There was greater variability in both scores among participants who did not change practice; this included all six instances of zero scores for both items.
Surgeons who did not change practice operated on a greater number of proximal humeral fractures (all types) and acute 'uncomplicated' displaced fractures of the proximal humerus involving the surgical neck (as eligible for the PROFHER trial) between April 2015 and March 2016 than those who did change practice (Table III). A low number of PROFHER-eligible fractures (frequency category 0 to 5 ) were operated on by $81.6 \%$ of surgeons who changed practice and $68.8 \%$ of surgeons who did not. None of the surgeons who changed practice operated on more than 15 PROFHER-eligible fractures.

The most popular surgical technique was fixation by locking plate (82.3\% overall). Participants who changed practice were more likely to use reverse shoulder arthroplasty (45.3\% versus $33.6 \%$ ) and less likely to use intramedullary nails ( $27.0 \%$ versus $38.3 \%$ ) than participants who did not change practice. 
Table III. Fracture treatment of participants who did/did not change practice because of the Proximal Fracture of the Humerus: Evaluation by Randomisation (PROFHER) trial

\begin{tabular}{|c|c|c|c|}
\hline & $\begin{array}{l}\text { Changed practice } \\
(n=137)\end{array}$ & $\begin{array}{l}\text { Did not change } \\
\text { practice }(n=128)\end{array}$ & Total $(n=265)$ \\
\hline \multicolumn{4}{|c|}{ Proximal humeral fractures (all types) operated in the last year, $\mathbf{n}(\%)$} \\
\hline 0 to 5 & $69(50.4)$ & $51(39.8)$ & $120(45.3)$ \\
\hline 6 to 10 & $34(24.8)$ & $28(21.9)$ & $62(23.4)$ \\
\hline 11 to 15 & $18(13.1)$ & $19(14.8)$ & $37(14.0)$ \\
\hline 16 to 20 & $12(8.8)$ & $15(11.7)$ & $27(10.2)$ \\
\hline $20+$ & 4 (2.9) & $14(10.9)$ & $18(6.8)$ \\
\hline Don't know & $0(0.0)$ & $1(0.8)$ & $1(0.4)$ \\
\hline Missing & 0 & 0 & 0 \\
\hline \multicolumn{4}{|l|}{ Surgical methods used, $\mathrm{n}(\%)^{*}$} \\
\hline Locking plate & $111(81.0)$ & $107(83.6)$ & $218(82.3)$ \\
\hline Intramedullary nail & $37(27.0)$ & $49(38.3)$ & $86(32.5)$ \\
\hline Hemiarthroplasty & $69(50.4)$ & $65(50.8)$ & $134(50.6)$ \\
\hline Reverse shoulder arthroplasty & $62(45.3)$ & $43(33.6)$ & $105(39.6)$ \\
\hline Other ${ }^{\dagger}$ & $8(5.8)$ & $6(4.7)$ & $14(5.3)$ \\
\hline Missing & 0 & 0 & 0 \\
\hline \multicolumn{4}{|c|}{ Fractures operated that were eligible for the PROFHER trial, n (\%) } \\
\hline 0 to 5 & $111(81.6)$ & $88(68.8)$ & $199(75.4)$ \\
\hline 6 to 10 & $13(9.6)$ & $17(13.3)$ & $30(11.4)$ \\
\hline 11 to 15 & $7(4.4)$ & $12(9.4)$ & $18(6.8)$ \\
\hline 16 to 20 & $0(0.0)$ & $3(2.3)$ & $3(1.1)$ \\
\hline $20+$ & $0(0.0)$ & $3(2.3)$ & $3(1.1)$ \\
\hline Don't know & $6(4.4)$ & $5(3.9)$ & $11(4.2)$ \\
\hline Missing & 1 & 0 & 1 \\
\hline \multicolumn{4}{|c|}{$\begin{array}{l}\text { Impact of the PROFHER cost-effectiveness findings on decision-making, } \\
\text { n (\%) }\end{array}$} \\
\hline Very influential & $8(6.0)$ & $2(1.6)$ & $10(3.9)$ \\
\hline Influential & $32(23.9)$ & $7(5.7)$ & $39(15.2)$ \\
\hline Somewhat influential & $47(35.1)$ & $47(38.2)$ & $94(36.6)$ \\
\hline Not at all influential & $47(35.1)$ & $67(54.5)$ & $114(44.4)$ \\
\hline Missing & 3 & 5 & 8 \\
\hline \multicolumn{4}{|c|}{ External changes to service delivery, n (\%) } \\
\hline Yes & $21(15.8)$ & $17(14.0)$ & $38(15.0)$ \\
\hline No & $112(84.2)$ & $104(86.0)$ & $216(85.0)$ \\
\hline Missing & 4 & 7 & 11 \\
\hline
\end{tabular}

*more than one response was possible for this question

†examples of responses to this question include: "Isolated tuberosity repair like cuff repair - sutures and anchors"; "intramedullary wires"; "intramedullary strut"; "T Plate"; and "LCDPlates"

Participants who changed their practice were more likely to consider the PROFHER cost-effectiveness findings as "very influential" or "influential" than in those who did not (29.9\% versus $7.3 \%)$.

A total of 38 respondents (15.0\%) indicated there had been external changes that had impacted on their current practice. Statements supplied by 37 of these indicated two main categories. The first related to changes to the delivery of trauma services, usually reflecting local availability of specialist colleagues (18 responses). The second (12 responses) indicated service pressures ("very busy trauma lists", bed and theatre availability issues) in various ways that impacted on surgical practice.

Qualitative results. The qualitative analysis of free-text data relating to change in practice was divided into groups of those participants who had or had not changed their practice because of PROFHER. The results of the fourth section of the survey, which explored wider factors affecting change in practice through free-text responses, are presented separately. In the following, we present the key emerging themes relating to these two aspects.
Respondents who had changed practice relating to PROFHER. All 137 respondents who indicated that they had changed practice provided further information about these changes through free-text responses. Most of these responses purely indicated a general move towards a more conservative approach. Where further information was offered, many highlighted how the PROFHER trial findings had given them greater confidence in making a decision to treat non-operatively (22 cases). Others suggested they had already been using conservative treatment for these patients (39 cases) and, in some instances, respondents commented that this had reduced concerns around liability as their practice was now evidence-based.

Several respondents indicated that they used the trial findings to inform a patient-centred approach, for example, through discussions of treatment options with patients in light of the trial findings (21 cases). Meanwhile, others commented on the need to review patients' need for surgery on a case-by-case basis (24 cases), with specific exclusion criteria from the PROFHER trial being important in these decisions (e.g. intra-articular 
extension or associated dislocation). Although not an exclusion criteria in the PROFHER trial, age was a consideration for some respondents, who generally stated that they only considered operating on younger patients. In some instances, respondents gave examples of how their surgical approach had altered over recent years; for example, a reduction in the use of locking plates was observed.

The influence of wider opinion, such as colleagues' change in practice, had apparently affected treatment decisions for some respondents. In some instances, respondents who were not specific shoulder specialists reported that the trial findings had led them to make fewer referrals to their specialist colleagues (nine cases). However, one respondent raised concerns that the trial findings may be misinterpreted by non-specialists, leading to reductions in referrals. Three respondents who had originally changed their practice in light of the PROFHER findings had reverted back to surgical management due to concerns around poorer outcomes from non-operative care; one indicating that "[the] study findings are not replicated in our population".

Of those giving specific reasons as to how the PROFHER trial findings had prompted a change in practice, many were encouraged to change practice due to there being no difference in patient outcomes across treatment groups, as well as the greater costs and potential for harm associated with surgery. Several respondents commented on the definitive nature of the trial findings, which had provided high-quality evidence to support these decisions. In a handful of cases where respondents had "partly changed" their practice, respondents were negative about the trial results, citing concerns around validity, sample size or preferring to rely on 'gut instinct' when treating patients.

Respondents who had not changed practice relating to PROFHER. The responses of the 111 respondents providing further information as to why they had not changed their practice as a result of the PROFHER trial fell into three broad categories: those indicating that the respondents were already managing these patients conservatively (43 cases); those critical about the study (33 cases); and heterogeneous comments that could not be grouped into an overarching theme (35 cases). Of note was that, similar to the respondents who had changed their practice, respondents commented that the PROFHER results had given greater support when considering non-surgical management (19 cases) and had additionally aided discussions with patients (eight cases).

Of those 43 respondents indicating they were already managing patients conservatively, similar comments emerged as to the group who had indicated their practice had changed in recent years (described earlier). For example, many commented on the greater confidence in treating non-operatively now, some with fewer referrals to specialist colleagues. Likewise, several stressed the need to review patients on a case-by-case basis, with surgery being offered for fractures excluded from the PROFHER trial in four cases. Of the 33 expressing some criticism or reservation with the PROFHER trial, there were ten respondents who were highly critical of the study ( $4 \%$ of total sample). These responses corresponded to very low scores, typically 0 , for the questionnaire items exploring perceived validity and applicability. Critical comments were mostly specific in nature, airing concerns about potential sources of bias such as selection bias (e.g. surgeons' ability to exclude those patients with a clear indication for surgery), generalisability of the study findings (e.g. exclusion of complex fractures; lack of reverse shoulder arthroplasties), or concerns around study design (several relating to the pragmatic study design).

In terms of heterogeneous comments that were not grouped into one overarching theme, these comments related to respondents' continuing reliance on their own judgement and professional experience that did not reflect the results of PROFHER and consequently led to a tendency for preferring surgical management.

General thoughts about factors influencing change in practice. Section 4 of the survey prompted free-text responses to three questions relating to factors influencing practice, with an option for further comments (Fig. 2). This optional section was completed by 136 respondents (51.1\%); more of whom changed practice $(56.2 \%, 77$ of 137) than did not (47.7\%, 59 of 129).

The need for an evidence base was the predominant response (110 cases) to the question asking "what factors are likely to influence your decision to change surgical practice?" The surgeons' own experience (30 cases) and peers' opinions (19 cases) were also expressed as important drivers for changing practice, with costs, choice of outcome measure used in research and needs of patients, including safety, featuring to lesser extents. For the second question, the majority considered that "practising EBM was becoming the norm in orthopaedic surgery", either fully (52 cases), partly (15 cases) or at least on the way (23 cases), with several respondents pointing to limitations in the available evidence (17 cases). A total of 20 respondents considered that it was not the norm.

Several themes arose from the responses on what the respondent's specialist society (e.g. BESS, BOA, British Society for Surgery of the Hand, Royal College of Surgeons) could do to guide change in their practice. One strong theme was the production of evidence-based guidelines, guidance (including standards) and summaries (including evidence updates and reviews) for information, to help and support decision-making (46 cases). This often linked with dissemination (24 cases), including at meetings in several cases. Several emphasised the need for critical appraisal. A second strong theme was society 
support, promotion, funding, and dissemination of research, mainly multicentre RCTs (29 cases), but also of other research that included epidemiology of conditions, national registries and identifying key clinical questions. Several directly endorsed the current activities, often specifically the support for large RCTs, of their specialist society (19 cases). There were a few exceptions, including those stressing the need to avoid the influence of industry.

Finally, when given the option of recording further comments, responses re-iterated the themes that had emerged mostly around the importance of the need for an evidence base and support for PROFHER, but also reservations about the limitations of the current evidence.

\section{Discussion}

This survey, which includes complete responses from 265 orthopaedic and trauma surgeons who are currently treating patients with proximal humerus fracture, confirms that the findings of the PROFHER trial have been well disseminated and have been considered by the United Kingdom's orthopaedic community in terms of their clinical practice. Around half (137) of these respondents had changed practice to various extents because of PROFHER, principally in that they treated fewer PROFHEReligible fractures surgically. A third (43) of the 128 respondents who had not changed practice were already managing patients consistent with the PROFHER findings, thus primarily non-operatively. The data suggest some differences in characteristics of the surgeons who did or did not change practice because of PROFHER; most notably those who changed practice were younger, worked in a trauma unit rather than a major trauma centre and were shoulder surgeons. This group gave higher scores when assessing validity and applicability of PROFHER. In contrast, a quarter of the non-changers were critical, sometimes emphatically, of PROFHER. The strongest message from the 136 participants who completed the section on general factors influencing change in practice was the endorsement of evidence-based orthopaedic practice.

This large, anonymous survey obtains both quantitative and qualitative data from the two main professional societies of surgeons operating on these fractures in the United Kingdom. The lack of the key denominator (all United Kingdom surgeons who were treating proximal humeral fractures at the time of the survey) means we cannot provide a response rate. Of note, however, is that the 144 shoulder surgeons who participated in the survey are likely to feature among the 550 shoulder and elbow surgeon members of BESS in 2017. Secondly, our analysis is based on data from 83.6\% (265 of 317 accesses) of those who accessed the website. Exploring possible response bias, we find that 39 respondents (15\%) had participated in the PROFHER trial (equating to
$45 \%$ of 87 named surgeons who assessed eligibility and including nine Pls of 35 recruiting sites). ${ }^{10}$ There was, therefore, a substantial response to the survey beyond those surgeons who were actively involved in the trial. Notably, whether surgeons participated or not in PROFHER did not influence the proportion who did and did not change practice.

Encouragingly, with the 265 orthopaedic and trauma surgeons responding to the survey, this emphasises the substantial interest in the findings of the trial beyond those involved in PROFHER. Another limitation of our survey is the lack of exact data to quantify the impact of PROFHER. Matching our experience with collecting this data in the trial itself, very few respondents gave an exact number of operations for proximal humeral fractures or for PROFHER eligible fractures they conducted over a one-year period. Nonetheless, the survey results supported a move towards non-surgical treatment because of the PROFHER results and, notably, a strong qualitative theme was the use of the PROFHER findings to support non-operative decisions, including helping discussions with patients.

This survey is one of various approaches to measuring impact of trials such as PROFHER. An intended repeat of the study by Dean et al ${ }^{11}$ that reviewed surgical practice in 11 United Kingdom hospitals between January 2014 and March 2015, thus before the Journal of the American Medical Association publication of PROFHER, ${ }^{1}$ will be valuable. However, as illustrated for the Distal Radius Acute Fracture Fixation Trial, using the date of main publication as the threshold for detecting change may be misleading. ${ }^{12}$ For PROFHER, several United Kingdom surgeons would have been aware of the main finding in the year before this as a result of presentations at meetings. Further complexity arises from evolving practice, crucially the increasing use of reverse shoulder arthroplasty (RSA) for more complex types of these fractures in older people corroborated in this survey and elsewhere. ${ }^{11}$ This has prompted the proposal for 'PROFHER-2', another multicentre trial that aims to examine the role of RSA for 3 and 4 part fractures in older patients.

The key finding from this survey is that PROFHER, a large, publicly funded, pragmatic randomised trial, has had an impact on surgeons' clinical practice, both changing it and underpinning existing non-operative practice. A potentially more important finding of the survey is the endorsement of evidence-based practice, and the active engagement of orthopaedic surgeons in multicentre clinical trials, which confirms a major phase change in the United Kingdom orthopaedic community. Indeed, the successful delivery by the orthopaedic surgeon community of PROFHER may be the most lasting impact of PROFHER, as it showed what is possible in terms of both the need for future research and the advancement of surgical knowledge and patient care. 


\section{References}

1. Rangan A, Handoll H, Brealey S, et al. Surgical vs nonsurgical treatment of adults with displaced fractures of the proximal humerus: the PROFHER randomized clinical trial. JAMA 2015;313:1037-1047.

2. Corbacho B, Duarte A, Keding A, et al. Cost effectiveness of surgical versus non-surgical treatment of adults with displaced fractures of the proximal humerus: economic evaluation alongside the PROFHER trial. Bone Joint J 2016;98-B:152-159.

3. No authors listed. National Institute for Health and Care Excellence. Fractures (noncomplex): assessment and management: NICE guideline. http://www.nice.org.uk/ guidance/ng38 (date last accessed 14 June 2017).

4. Palvanen M, Kannus $\mathbf{P}$, Niemi S, Parkkari J. Update in the epidemiology of proximal humeral fractures. Clin Orthop Relat Res 2006;442:87-92.

5. Court-Brown CM, Caesar B. Epidemiology of adult fractures: A review. Injury 2006;37:691-697.

6. Bell JE, Leung BC, Spratt KF, et al. Trends and variation in incidence, surgical treatment, and repeat surgery of proximal humeral fractures in the elderly. $J$ Bone Joint Surg [Am]2011;93-A:121-131.

7. Polinder S, lordens GI, Panneman MJ, et al. Trends in incidence and costs of injuries to the shoulder, arm and wrist in The Netherlands between 1986 and 2008 BMC Public Health 2013:13:531.

8. Lamond D, Crow R, Chase J, Doggen K, Swinkels M. Information sources used in decision making: considerations for simulation development. Int J Nurs Stud 1996;33:47-57.

9. Ritchie J, Spencer L. Qualitative data analysis for applied policy research. In: Bryman A, Burgess R, eds. In: Analyzing qualitative data. London: Routledge, 1994.

10. Handoll H, Brealey S, Rangan A, et al. The ProFHER (PROximal Fracture of the Humerus: evaluation by Randomisation) trial - a pragmatic multicentre randomised controlled trial evaluating the clinical effectiveness and cost-effectiveness of surgical compared with non-surgical treatment for proximal fracture of the humerus in adults. Health Technol Assess 2015;19:1-280.

11. Dean BJ, Jones LD, Palmer AJ, et al. A review of current surgical practice in the operative treatment of proximal humeral fractures: does the PROFHER trial demonstrate a need for change? Bone Joint Res 2016;5:178-184

12. Costa ML, Jameson SS, Reed MR. Do large pragmatic randomised trials change clinical practice?: assessing the impact of the Distal Radius Acute Fracture Fixation Trial (DRAFFT). Bone Joint J 2016;98-B:410-413
Funding Statement

This work, as part of the ProFHER trial funding, was supported by the Nationa Institute for Health Research (NIHR) Health Technology Assessment Programm (Project number: 06/404/53). The views and opinions expressed are those of the authors and do not necessarily reflect those of the UK National Health Service, the UK National Institute for Health Research, or the UK Department of Health.

Author Contribution

L. Jefferson: Conceiving the study, Preparing the electronic questionnaire, Qualitative analysis, Writing and reviewing the article.

- S. Brealey: Conceiving the study, Preparing the electronic questionnaire, Qualitative analysis, Writing and reviewing the article.

- H. Handoll: Conceived the study, Qualitative analysis, Writing and reviewing the article.

A. Keding: Designing the questionnaire tool, Statistical analysis, Writing and reviewing the article.

L. Kottam: Conducting the pilot, Writing and reviewing the article.

I. Sbizzera: Statistical analysis, Writing and reviewing the article.

A. Rangan: Conceiving the study, Organising the distribution, Writing and review ing the article.

Acknowledgements

We thank the surgeons who participated in completing the online survey and the orthopaedic surgeons who completed the 'think aloud' process (Mr Rajesh Nanda, Mr Jaime Candal-Couto, Mr Amit Bidwai, Mr Andrew Brooksbank, Mr Philip Ahrens and $\mathrm{Mr}$ Hagen Jahnich). We are grateful to the British Orthopaedic Association and the British Elbow and Shoulder Society for distributing this online survey and will share with them our findings about the uptake of evidence-based medicine and potential role for specialist societies in disseminating research evidence. We are grateful to Miss Katherine Chatterton who assisted with the development of the online survey using the Qualtrics software.

Ethical approval was given by the Department of Health Sciences Research Governance Committee, University of York, prior to dissemination of the survey.

- Dr Laura Jefferson is study guarantor. All authors had access to the data in the study and can take responsibility for the integrity and accuracy of the data analysis.

Conflicts of Interest Statement

Erofessor Amar Rangan reports grants from De Puy Ltd and JRI Ltd; both are outside the submitted work. None of these influenced the trial or this report.

(c) 2017 Jefferson et al. This is an open-access article distributed under the term of the Creative Commons Attributions licence (CC-BY-NC), which permits unrestricted use, distribution, and reproduction in any medium, but not for commercial gain, provided the original author and source are credited. 